\title{
Kinematics of coronal rain in a transversely oscillating loop: Ponderomotive force and rain-excited oscillations
}

\author{
E. Verwichte ${ }^{1}$, P. Antolin ${ }^{2}$, G. Rowlands ${ }^{1}$, P. Kohutova ${ }^{1}$, and T. Neukirch ${ }^{2}$ \\ 1 Department of Physics, University of Warwick, Coventry CV4 7AL, UK \\ e-mail: Erwin.Verwichte@warwick.ac.uk \\ 2 School of Mathematics and Statistics, University of St. Andrews, St Andrews KY16 9SS, UK \\ Received 1 September 2016 / Accepted 2 November 2016
}

\begin{abstract}
Context. Coronal rain is composed of cool dense blobs that form in solar coronal loops and are a manifestation of catastrophic cooling linked to thermal instability. Once formed, rain falls towards the solar surface at sub-ballistic speeds, which is not well understood. Pressure forces seem to be the prime candidate to explain this. In many observations rain is accompanied by transverse oscillations and the interaction between rain and these oscillations needs to be explored.

Aims. Therefore, an alternative kinematic model for coronal rain kinematics in transversely oscillating loops is developed to understand the physical nature of the observed sub-ballistic falling motion of rain. This model explicitly explores the role of the ponderomotive force arising from the transverse oscillation on the rain motion and the capacity of rain to excite wave motion.

Methods. An analytical model is presented that describes a rain blob guided by the coronal magnetic field supporting a onedimensional shear Alfvén wave as a point mass on an oscillating string. The model includes gravity and the ponderomotive force from the oscillation acting on the mass and the inertia of the mass acting on the oscillation.

Results. The kinematics of rain in the limit of negligible rain mass are explored and falling and trapped regimes are found, depending on wave amplitude. In the trapped regime for the fundamental mode, the rain blob bounces back and forth around the loop top at a long period that is inversely proportional to the oscillation amplitude. The model is compared with several observational rain studies, including one in-depth comparison with an observation that shows rain with up-and-down bobbing motion. The role of rain inertia in exciting transverse oscillations is explored in inclined loops.

Conclusions. It is found that the model requires displacement amplitudes of the transverse oscillation that are typically an order of magnitude larger than observed to explain the measured sub-ballistic motion of the rain. Therefore, it is concluded that the ponderomotive force is not the primary reason for understanding sub-ballistic motion, but it plays a role in cases of large loop oscillations. The appearance of rain causes the excitation of small-amplitude transverse oscillations that may explain observed events and provide a seismological tool to measure rain mass.
\end{abstract}

Key words. Sun: corona - Sun: oscillations - Sun: activity - magnetohydrodynamics (MHD) - methods: analytical - waves

\section{Introduction}

Coronal rain are cool dense blobs that form in warm active region loops and fall along the guiding magnetic field to the solar surface (Kawaguchi 1970; Leroy 1972; Athay et al. 1980; Schrijver 2001; De Groof et al. 2004; de Groof et al. 2005; Vashalomidze et al. 2015). They are traditionally observed in chromospheric lines, such as $\mathrm{H}-\alpha$ or $\mathrm{Ca}$ II H, as well as in transition region lines. But they can also be detected in absorption in the extreme ultraviolet (EUV). It is believed that coronal rain is a manifestation of catastrophic cooling of coronal loop plasma in response to localised heating near the loop footpoints (e.g. Müller et al. 2005; Klimchuk et al. 2008; Antolin et al. 2010). As such it is an important characteristic of the coronal heating process.

Using the Solar Optical Telescope (SOT) on board the Hinode satellite (Tsuneta et al. 2008) and the CRisp Imaging SpectroPolarimeter at the Swedish $1 \mathrm{~m}$ Solar Telescope (Scharmer et al. 2003, 2008), Antolin et al. (2010), Antolin \& Rouppe van der Voort (2012), Antolin et al. (2015) showed, with on-disk and limb observations, that coronal rain is a common occurrence in warm active region loops. Coronal rain is dynamic with lifetimes of a few minutes, widths of several hundred $\mathrm{km}$ and lengths of a few Mm. The blobs become more thinner and elongated towards the footpoints. Their small sizes makes them particularly useful for probing the local magnetic field structure (Antolin \& Rouppe van der Voort 2012). Coronal rain has been measured with temperatures ranging between $3000 \mathrm{~K}$ to $150000 \mathrm{~K}$ (Levine \& Withbroe 1977; Müller et al. 2005) and is expected from simulations to have a density of $10^{16}-10^{18} \mathrm{~m}^{-3}$ (Antolin et al. 2010; Luna et al. 2012; Fang et al. 2013, 2015); this value is confirmed by observations (Antolin et al. 2015). The downflow speed of the rain ranges between 30 up to supersonic speeds of $200 \mathrm{~km} \mathrm{~s}^{-1}$ with an average of $60-70 \mathrm{~km} \mathrm{~s}^{-1}$ (Ahn et al. 2014; Kleint et al. 2014; Antolin et al. 2015; Straus et al. 2015).

The downwards acceleration is usually of the order of $80 \pm$ $30 \mathrm{~m} \mathrm{~s}^{-2}$ (Antolin \& Rouppe van der Voort 2012), which is substantially sub-ballistic, even when taking the loop geometry and line of sight into account. This would mean that other forces act upwards along the loop against gravity. The prime candidate force arises from the coronal plasma below the rain blob that is being compressed as the rain falls towards the surface (Müller et al. 2003; Oliver et al. 2014). The resulting drag causes the loop cross section to expand radially outwards below the 
blob. If the magnetic field is strong enough, the resulting radial magnetic tension restoring force acts to counteract this and may even cause the rain blob to rebound several times near the footpoints (Mackay \& Galsgaard 2001).

Antolin \& Verwichte (2011) presented an observational study with Hinode/SOT in In $\mathrm{Ca}$ II $\mathrm{H}$ in which multiple coronal rain blobs are seen to oscillate transversely in phase. The oscillation periods lie between $100 \mathrm{~s}$ and $200 \mathrm{~s}$ with displacement amplitudes of the order of $500 \mathrm{~km}$ or less. It was concluded that the rain traces out a kink (Alfvénic) wave, either standing or running, supported by the whole coronal loop plasma (Nakariakov et al. 1999; Van Doorsselaere et al. 2008a; Goossens et al. 2009). For this event, the coronal rain acceleration, which has a value of $56 \mathrm{~m} \mathrm{~s}^{-2}$ is also significantly sub-ballistic. Kohutova \& Verwichte (2016) detected two different regimes of transverse oscillations traced by the rain in a rain event observed by IRIS (De Pontieu et al. 2014): small-scale persistent oscillations driven by a continuously operating process and localised large-scale oscillations excited by a transient mechanism. Transverse oscillations of coronal loops have frequently been observed in the EUV (e.g. Aschwanden et al. 1999, 2002; Wang \& Solanki 2004; van Doorsselaere et al. 2008b; Verwichte et al. 2009; Aschwanden \& Schrijver 2011; White \& Verwichte 2012). Large-amplitude oscillations with displacements of several megameters are often excited impulsively by a nearby flare or CME (e.g. Aschwanden et al. 2002; Wang \& Solanki 2004; De Moortel \& Brady 2007; Van Doorsselaere et al. 2007; Verwichte et al. 2009, 2010; Mrozek 2011; White \& Verwichte 2012), or directly by the reconnection process (White et al. 2012, 2013). The excitation mechanism of small-amplitude oscillations is less clear. Verwichte et al. (2004) reported oscillations in a post-flare loop arcade that were driven by the motions of a nearby partially erupting prominence. Decay-less or even growing continuous quasi-periodic oscillations have been reported and were associated with a lower atmosphere driver or nearby CME (Wang et al. 2012; Nisticò et al. 2013; Anfinogentov et al. 2015). Smallamplitude running transverse waves guided along the loops have been reported by Tomczyk et al. (2007), McIntosh et al. (2011), Threlfall et al. (2013). Small-amplitude oscillations are also often seen in coronal rain (Antolin \& Verwichte 2011; Kohutova \& Verwichte 2016). The potential of transverse waves for seismological probing of the coronal structure and magnetic field has been explored extensively (e.g. Nakariakov \& Ofman 2001; Andries et al. 2009; Verth et al. 2010; Arregui \& Asensio Ramos 2011; Goossens et al. 2012; Verwichte et al. 2013a,b; Pascoe et al. 2013).

Here we investigate the effect of the nonlinear ponderomotive force generated by a transverse oscillation (e.g. Allan 1993; Verwichte et al. 1999; Terradas \& Ofman 2004) on the acceleration of coronal rain and evaluate its dependence on amplitude. Furthermore, we study the role of coronal rain in exciting transverse oscillations. In magnetohydrodynamics (MHD) a common definition of the ponderomotive force is as the nonlinear component of the Lorentz force along the equilibrium magnetic field resulting from the magnetic field perturbation of an oscillation or wave. Allan \& Manuel (1996) demonstrated in a magnetospheric context that the ponderomotive force from MHD waves may transport plasma over large distances along the equilibrium magnetic field. They also suggested, without detail, that the ponderomotive force from Alfvén waves can be compared by analogy to the frictionless motion of beads along a transversely oscillating, stretched string. Verwichte (1999) has shown that up to third order in the normalised oscillation amplitude the modelling of the transverse wave using this mechanical analogue or using MHD are in agreement. We develop a 1.5 dimensional nonlinear mechanical model, which allows us to easily resolve both coronal blob kinematics and the transverse oscillation.

The paper is structured as follows. In Sect. 2 we present the mechanical model and derive the coupled system of differential equations for the rain kinematics and transverse wave field. In Sect. 3 we study the rain kinematics in the presence of a single oscillation harmonic under the assumption of negligible rain mass. In Sect. 4 we apply the model to an example observation set of coronal rain from Hinode/SOT. In Sect. 5 we model the excitation of the transverse oscillation by rain in an inclined loop. Finally, in Sect. 6 we discuss our findings.

\section{Mechanical model}

We model coronal rain as a single blob of mass $m$ that is frozen in to the magnetic field line as a bead attached to a string. The magnetic field line has a semi-circular shape with a major radius $R$ and loop length $L=\pi R$. We neglect the transverse structuring for simplicity. The position of the blob is given by its position parallel to the magnetic field, $s_{\mathrm{p}}(t)$, and normal to the loop plane, $x_{\mathrm{p}}(t)$. We include gravity, which along the field line is of the form $g(s)=g_{\odot} \cos (s / R)$, where $s$ is the independent coordinate along the loop. The displacement of the loop in the direction normal to the loop plane is given by $\xi(s, t)$.

The Langrangian for this system is given by

$$
\begin{aligned}
\mathcal{L}= & \int_{0}^{L} S \mathrm{~d} s\left[\frac{\rho}{2}\left(\frac{\partial \xi}{\partial t}\right)^{2}-\frac{B^{2}}{2 \mu_{0}}\left(\frac{\partial \xi}{\partial s}\right)^{2}\right] \\
& +\frac{m}{2}\left[\left(\frac{\mathrm{d} s_{\mathrm{p}}}{\mathrm{d} t}\right)^{2}+\left(\frac{\mathrm{d} x_{\mathrm{p}}}{\mathrm{d} t}\right)^{2}\right]-m \int_{0}^{s_{\mathrm{p}}} g\left(s^{\prime}\right) \mathrm{d} s^{\prime},
\end{aligned}
$$

where $S$ is a constant loop cross section, $\rho$ is the mass density of the coronal plasma, $B$ is a uniform magnetic field strength, and $\mu_{0}$ is the permeability of free space. The integral term is the difference between the kinetic energy and potential energy of the transverse wave motion associated with the magnetic tension force. The second and third terms are the difference between the kinetic energy and gravitational potential energy of the rain blob. A constraint is added to ensure the blob remains on the field line, i.e.

$x_{\mathrm{p}}=\int_{0}^{L} \mathrm{~d} s \delta\left(s-s_{\mathrm{p}}\right) \xi(s, t)$.

This model is essentially one-dimensional with propagation only along the magnetic field line. The fast magneto-acoustic and Alfvén waves are degenerate and both describe a transverse wave. Because we do not include transverse loop structuring, the typical wave phase speed is the Alfvén speed instead of the kink speed. For simplicity, we assume that there is no mass flux into or out of the loop. Then, the total loop mass $M=m+L S \rho$ is a constant. We introduce the dimensionless parameter

$\mu=\frac{m}{L S \rho}=\frac{m}{M}\left(1-\frac{m}{M}\right)^{-1}$,

which ranges from zero (no coronal rain), to unity (half rain, half coronal plasma), to infinity (no coronal plasma). We find $m / M=0.2$ and $\mu=0.25$ for coronal rain that is ten times denser than the coronal plasma and extending in length over $2 \mathrm{Mm}$ in a $100 \mathrm{Mm}$ loop (Antolin et al. 2010). If we independently include an additional reduction in cross-sectional area for rain by 
a factor of say 36 to take the observed transverse fine structure into account (Antolin \& Rouppe van der Voort 2012), we find a much lower value of $m / M \approx \mu \approx 0.01$. On the other hand we may also consider modelling a complete rain shower (Antolin \& Rouppe van der Voort 2012) event as a whole, for which the total mass ratio may be as high as unity (Antolin et al. 2015).

The equations of motion describing the transverse oscillation and the blob motion follow from minimising the functional

$I=\int_{t_{1}}^{t_{2}} \mathrm{~d} t\left[\mathcal{L}+\lambda\left(x_{\mathrm{p}}-\int_{0}^{\pi R} \mathrm{~d} s \delta\left(s-s_{\mathrm{p}}\right) \xi(s, t)\right)\right]$,

with respect to the dependent variables where $\lambda$ is a Lagrangian multiplier. We also add a Rayleigh-type dissipative functional of the form

$\mathcal{D}=-\frac{1}{\tau} \int_{0}^{\pi R} S \mathrm{~d} s \rho\left(\frac{\partial \xi}{\partial t}\right)^{2}$,

where $\tau$ represents a typical time over which transverse oscillation damp due to, for example, resonant absorption (Ruderman \& Roberts 2002). This leads to the equations

$\frac{\mathrm{d}^{2} s_{\mathrm{p}}}{\mathrm{d} t^{2}}=-\left.\frac{g(s)+\frac{\mathrm{d}^{2} \xi}{\mathrm{d} t^{2}} \frac{\partial \xi}{\partial s}}{1+\left(\frac{\partial \xi}{\partial s}\right)^{2}}\right|_{s=s_{\mathrm{p}}}$,
$\left[\frac{\partial^{2}}{\partial t^{2}}+\frac{2}{\tau} \frac{\partial}{\partial t}-V_{\mathrm{A}}^{2} \frac{\partial^{2}}{\partial s^{2}}\right] \xi=-\left.\mu L \frac{\mathrm{d}^{2} \xi}{\mathrm{d} t^{2}}\right|_{s=s_{\mathrm{p}}} \delta\left(s-s_{\mathrm{p}}\right)$,

and

$\frac{\mathrm{d}^{2} \xi}{\mathrm{d} t^{2}}=\frac{\partial^{2} \xi}{\partial t^{2}}+2 \frac{\partial^{2} \xi}{\partial t \partial s}\left(\frac{\mathrm{d} s_{\mathrm{p}}}{\mathrm{d} t}\right)+\frac{\partial^{2} \xi}{\partial s^{2}}\left(\frac{\mathrm{d} s_{\mathrm{p}}}{\mathrm{d} t}\right)^{2}$.

The Alfvén speed of the coronal plasma is given by

$V_{\mathrm{A}}=\frac{B}{\sqrt{\mu_{0} \rho}}=B \sqrt{\frac{S \pi R}{\mu_{0} M}} \sqrt{\frac{M}{M-m}}=V_{\mathrm{A} 0} \sqrt{1+\mu}$.

The right-hand side of Eq. (6) shows that a coronal rain blob feels two competing forces: a downwards pointing gravity and a ponderomotive force from the wave field.

If we assume that the blob is light so that $\mu \ll 1$, then the wave is not modified by the presence of the blob and is readily described by the solution

$\xi(s, t)=\frac{a}{k} \mathrm{e}^{-t / \tau} \sin (k s) \cos (\omega t)$,

where $k=n / R, \omega \approx V_{\mathrm{A}} k$ for weak damping and $n$ is an integer. The equation of motion of the blob itself is then described by

$\frac{\mathrm{d}^{2} s}{\mathrm{~d} t^{2}}=a_{0} \frac{\omega^{2}}{k}+a_{1} \omega \frac{\mathrm{d} s}{\mathrm{~d} t}+a_{2} k\left(\frac{\mathrm{d} s}{\mathrm{~d} t}\right)^{2}$,

where the subscript " $p$ " has been dropped for simplicity and where

$a_{0}(s, t)=-f\left[\frac{1}{2} a^{2} \sin (2 k s) \frac{T}{\omega^{2}} \frac{\mathrm{d}^{2} T}{\mathrm{~d} t^{2}}+\alpha^{2} \cos \left(\frac{s}{R}\right)\right]$,

$a_{1}(s, t)=-2 f a^{2} \cos ^{2}(k s) \frac{T}{\omega} \frac{\mathrm{d} T}{\mathrm{~d} t}$,

$a_{2}(s, t)=\frac{1}{2} a^{2} f \sin (2 k s) T^{2}$,

$f(s, t)=\left[1+a^{2} \cos ^{2}(k s) T^{2}\right]^{-1}$,

$T(t)=\mathrm{e}^{-t / \tau} \cos (\omega t)$, with

$\alpha=\frac{\sqrt{k g_{\odot}}}{\omega}$.

For typical coronal conditions, with $g_{\odot}=274 \mathrm{~m} \mathrm{~s}^{-2}$, and assuming wave period and wavelengths of $300 \mathrm{~s}$ and $200 \mathrm{Mm}$, respectively, we find $\alpha=0.08$. Thus, an assumption of a regime of $\alpha \ll 1$ is reasonable. To leading order in $a$, the term $a_{0}$ has a contribution to the blob acceleration from the transverse oscillation that does not vanish when averaging over a period of oscillation in the limit of no damping. This term represents a secular ponderomotive acceleration acting on the blob.

\section{Coronal rain kinematics in presence of transverse oscillation}

For our model, we consider the stationary points and their stability in the limit of $\tau \rightarrow \infty$. There is always a stationary point at the loop top, i.e. $s=\pi R / 2$, where the gravitational acceleration along the field line is zero. In the absence of an oscillation $(a=0)$ any small displacement from the loop top would result in the blob falling in free-fall to the solar surface. However, if the oscillation amplitude is large enough the ponderomotive force can balance gravity and the blob may remain suspended at or oscillate around the loop top. We consider that $a, \alpha \sim O(\epsilon)$ where $\epsilon \ll 1$. The kinematics of the blob is modelled by the introduction of two timescales, i.e. $t_{0}=t$ and $t_{1}=\epsilon t$, and an expansion of $s=s_{0}\left(t_{0}, t_{1}\right)+\epsilon^{2} s_{2}\left(t_{0}, t_{1}\right)$ (Nayfeh 2004). Equation (11) then reduces to orders $O(1)$ and $O(\epsilon)$ to the conditions

$\frac{\partial^{2} s_{0}}{\partial t_{0}^{2}}=0, \frac{\partial^{2} s_{0}}{\partial t_{0} \partial t_{1}}=0 \Rightarrow s_{0}=s_{0}\left(t_{1}\right)$,

which shows that $s_{0}$ is only a function of $t_{1}$. The terms in Eq. (11) of $O\left(\epsilon^{2}\right)$ reduce to

$\frac{\partial^{2} s_{2}}{\partial t_{0}^{2}}-\frac{a^{2} \omega^{2}}{4 k} \sin \left(2 k s_{0}\right) \cos \left(2 \omega t_{0}\right)=-\frac{\mathrm{d}^{2} s_{0}}{\mathrm{~d} t_{1}^{2}}-\frac{\partial U}{\partial s_{0}}$,

where

$U\left(s_{0}\right)=\frac{a^{2} V_{\mathrm{A}}^{2}}{8}\left[\cos \left(2 k s_{0}\right)+\frac{8 n \alpha^{2}}{a^{2}} \sin \left(\frac{s_{0}}{R}\right)\right]$.

Because $U$ is not a function of time, the last term of Eq. (15) would lead to a secularly growing solution of $s_{2}$, unless the righthand side of Eq. (15) is zero. This leads to an evolutionary equation for $s_{0}$ with respect to slow time $t_{1}$ as follows:

$\frac{\mathrm{d}^{2} s_{0}}{\mathrm{~d} t_{1}^{2}}+\frac{\partial U}{\partial s_{0}}=0$

which may be integrated to an energy balance equation,

$\frac{1}{2}\left(\frac{\mathrm{d} s_{0}}{\mathrm{~d} t_{1}}\right)^{2}+U\left(s_{0}\right)=\mathcal{E}$,

where $\mathcal{E}$ is a constant of integration that is determined by initial conditions. The function $U$ fulfils the role of a potential. The solution of Eq. (15) is then

$s_{2}=-\frac{a^{2}}{16 k} \sin \left(2 k s_{0}\right) \cos \left(2 \omega t_{0}\right)$, 
which shows that on top of the slow large-scale dynamics of the blob there are small oscillations superimposed at half the oscillation period of the loop.

To understand the blob kinematics we study the topology of Eq. (18) in the phase space of $s_{0}$ and $\frac{\mathrm{d} s_{0}}{\mathrm{~d} \tau}$. Critical points exist where the potential is extremal, i.e. $\frac{\partial U}{\partial s_{0}}=0$,

$\sin \left(\frac{2 n s_{0}}{R}\right)=\left(\frac{2 \alpha}{a}\right)^{2} \cos \left(\frac{s_{0}}{R}\right)$,

or using a binomial expansion for the sine function on the lefthand side,

$$
\begin{aligned}
& {\left[\sum_{j=1}^{n}\left(\begin{array}{c}
2 n \\
2 j-1
\end{array}\right)(-1)^{j} \sin ^{2 j-1}\left(\frac{s_{0}}{R}\right) \cos ^{2(n-j)}\left(\frac{s_{0}}{R}\right)+\left(\frac{2 \alpha}{a}\right)^{2}\right]} \\
& \quad \times \cos \left(\frac{s_{0}}{R}\right)=0 .
\end{aligned}
$$

This equation confirms that the loop top, i.e. $s_{0}=\pi R / 2$, is always a critical point. In addition there are up to $2 n$ more critical points. The nature of the critical points is determined by the sign of the second derivative of the potential,

$\frac{\partial^{2} U}{\partial s_{0}^{2}}=-\frac{a^{2} \omega^{2}}{2}\left[\cos \left(2 k s_{0}\right)+\frac{2 \alpha^{2}}{n a^{2}} \sin \left(\frac{s_{0}}{R}\right)\right]$,

with a positive (negative) sign corresponding to a centre (saddle point). The loop top is a centre only if $n$ is an odd integer and

$\left(\frac{\alpha}{a}\right)^{2}<\frac{n}{2} \Rightarrow a>a_{\text {crit }}=\frac{\sqrt{2 g_{\odot} R}}{V_{\mathrm{A}} n}$.

This condition is the minimum oscillation amplitude required to prevent the blob from falling. For typical coronal values of $R=50 \mathrm{Mm}$ and $V_{\mathrm{A}}=1000 \mathrm{~km} \mathrm{~s}^{-1}$, we find $a_{\text {crit }}=0.17 / n$, which corresponds to a loop displacement amplitude of $8 / n \mathrm{Mm}$. Oscillations with such amplitudes have been observed by e.g. Hershaw et al. (2011). For the fundamental mode $(n=1)$, two other critical points exist if $a>a_{\text {crit }}$,

$s_{0}=R \arcsin \left(\frac{4 \alpha^{2}}{a^{2}}\right), s_{0}=R\left[\pi-\arcsin \left(\frac{4 \alpha^{2}}{a^{2}}\right)\right]$,

which can easily be verified to be saddle points. The phase diagram for $n=1$ for the cases where $a<a_{\text {crit }}$ and $a>a_{\text {crit }}$ are illustrated in Fig. 1.

Between the two saddle points, the blob may oscillate. The period of oscillation is found by expanding Eq. (17) around the loop top with $s_{0}=\pi R / 2+\delta s$,

$\frac{\mathrm{d}^{2} \delta s}{\mathrm{~d} t_{1}^{2}}=-\Omega^{2} \delta s$,

where

$\Omega^{2}=\left.\frac{\partial^{2} U}{\partial s_{0}^{2}}\right|_{s_{0}=\pi R / 2}=\frac{1}{2} a^{2} \omega^{2}-\frac{g_{\odot}}{R}=\frac{1}{2} \omega^{2}\left(a^{2}-a_{\text {crit }}^{2}\right)$.

For $a=0.2-0.5$, the same typical coronal values as before, and a transverse oscillation period of $300 \mathrm{~s}$ we find a blob period in the range $900-3500 \mathrm{~s}$. For fixed oscillation frequency $\omega, \Omega$ is independent of the wave number $n$. Also, the maximum blob displacement is $\delta s=R \arcsin \left(4 \alpha^{2} / a^{2}\right)$. The slow up-and-down oscillation of the blob is a clear signature of the ponderomotive
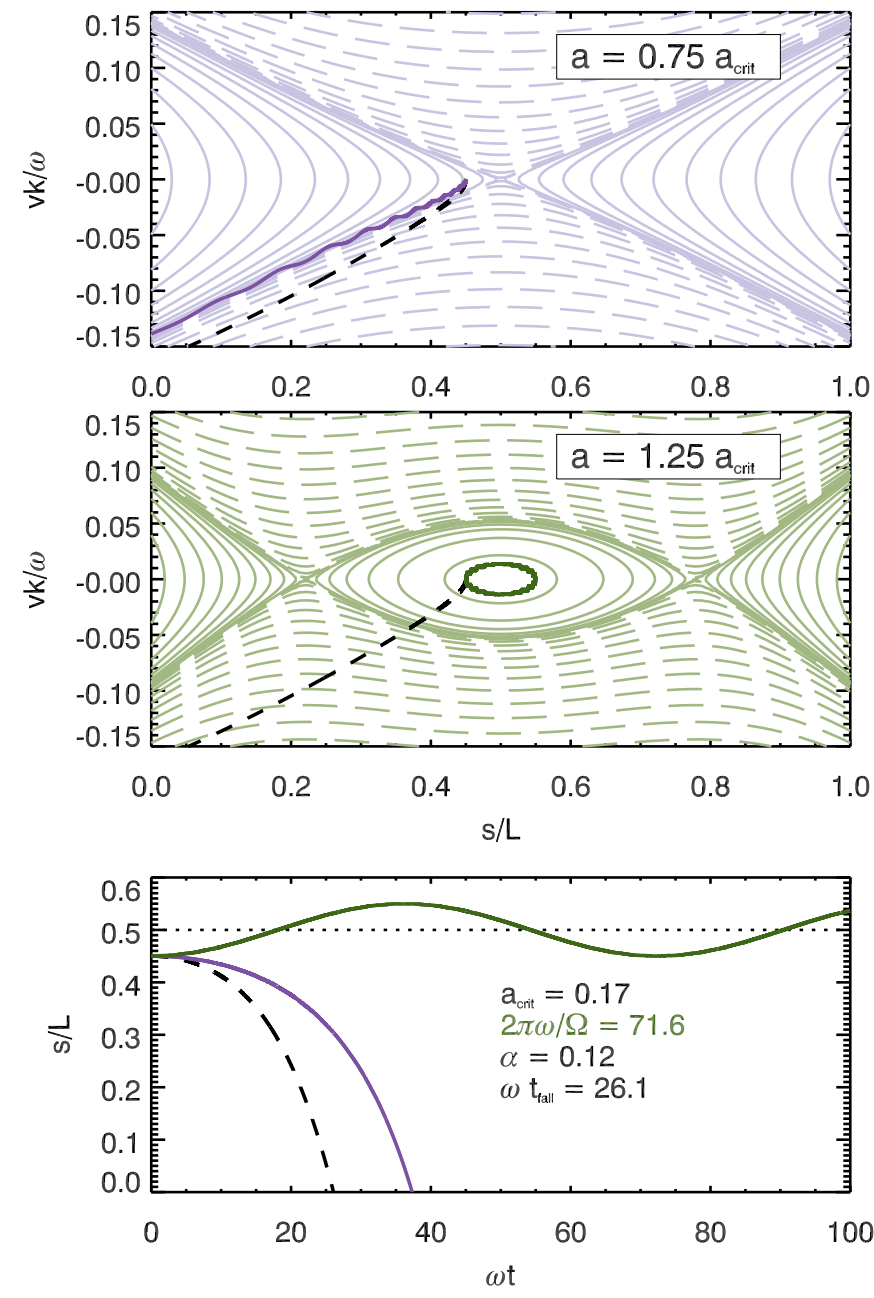

Fig. 1. Phase diagram associated with Eq. (17) and $n=1$, for $a<a_{\text {crit }}$ (top) and $a>a_{\text {crit }}$ (middle). The solid line is the path of a blob with initial position $s(0)=0.45 \mathrm{~L}$ and initial velocity $v(0)=-10^{-5} \omega / k$. The dashed line is the path of a blob with the same initial conditions in the absence of an oscillation $(a=0)$. The bottom panel shows the distance along loop of the blobs as a function of time for the three cases.

force acting on coronal rain blob in a coronal loop and is prescribed by the transverse loop oscillation amplitude and period.

For the first harmonic overtone $(n=2)$, the loop top is always a saddle point. However, there can exist centres near the maxima in the oscillation displacement halfway between footpoint and loop top. From Eq. (21), we obtain a third-order polynomial in $\sin \left(s_{0} / R\right)$,

$\sin ^{3}\left(\frac{s_{0}}{R}\right)-\frac{1}{2} \sin \left(\frac{s_{0}}{R}\right)+\frac{\alpha^{2}}{2 a^{2}}=0$

which has three real solutions (a saddle point at the loop top and a centre in each loop leg) only if the discriminant is negative, i.e.

$\left(\frac{\alpha}{a}\right)^{4}<\frac{2}{27} \Rightarrow a>a_{\text {crit }}=\left(\frac{3}{2}\right)^{3 / 4} \frac{\sqrt{g_{\odot} R}}{V_{\mathrm{A}}}$.

The value $a_{\text {crit }}$ found here is similar to that for the centre at the loop top for a fundamental mode. The location of the two centres in the loop legs requires solving the third-order polynomial. From considering the two extreme cases of $a=a_{\text {crit }}$ and $a \rightarrow \infty$, 

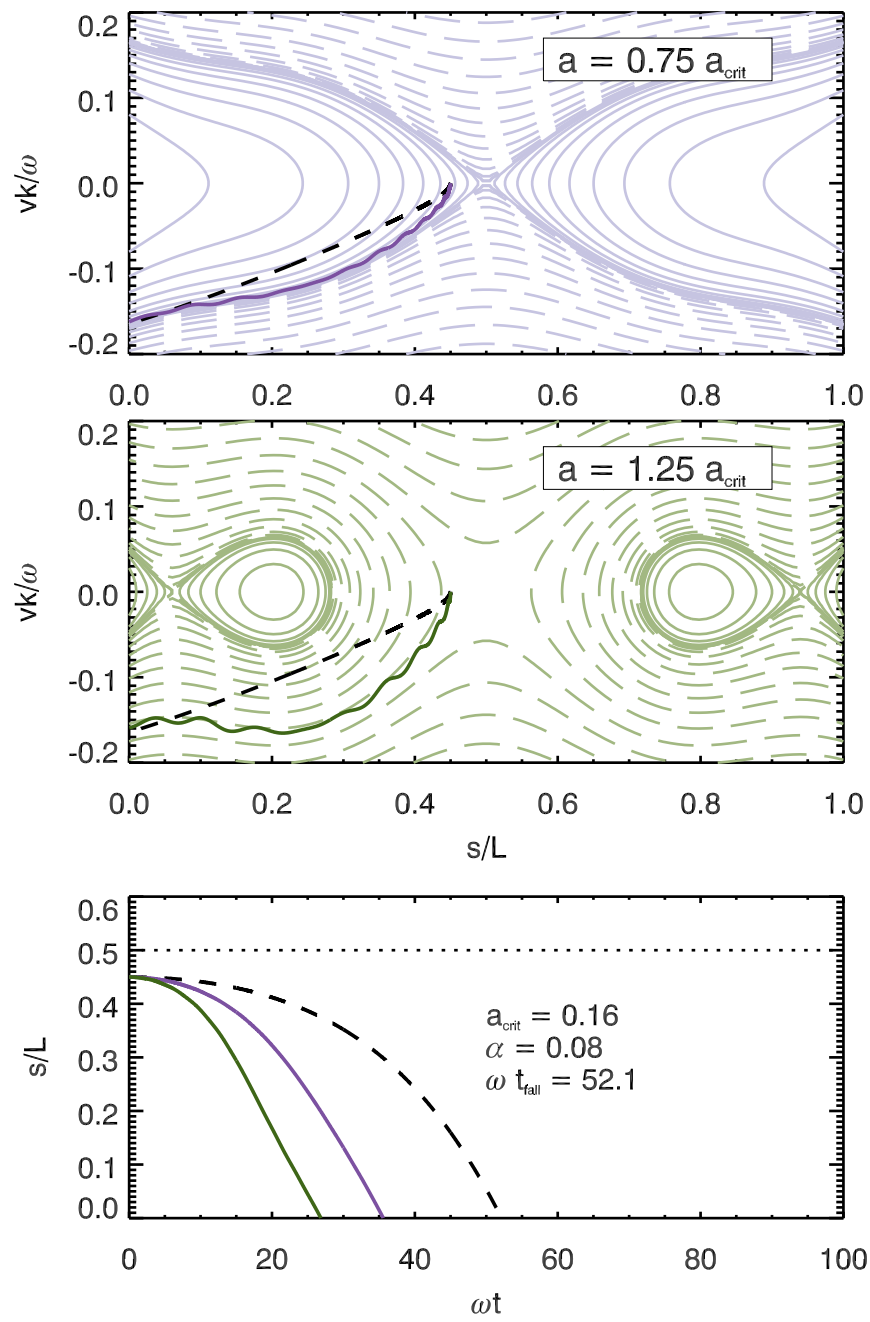

Fig. 2. Same as Fig. 1 for $n=2$. Because the mode frequency $\omega$ of the first overtone is twice that of the fundamental mode, the normalised ballistic fall time $\omega t_{\text {fall }}$ is double the value here compared with Fig. 1 whilst the actual time $t_{\text {fall }}$ remains the same.

we find that they are located within the intervals

$\arcsin \left(\frac{1}{\sqrt{6}}\right) \leq \frac{s_{0}}{R} \leq \frac{\pi}{4}, \frac{3 \pi}{4} \leq \frac{s_{0}}{R} \leq \pi-\arcsin \left(\frac{1}{\sqrt{6}}\right)$.

We integrate Eq. (11) numerically using an explicit Runge-Kutta method at fourth-order accuracy (RK4). Figures 1 and 2 illustrate the kinematics of a rain blob in a transverse loop oscillation for $\tau \rightarrow \infty$. For a fundamental transverse oscillation with $a<a_{\text {crit }}$, the blob is seen falling to the photosphere at a sub-ballistic rate. If $a>a_{\text {crit }}$ and the initial speed is not large, then the ponderomotive force is strong enough to keep the blob oscillating around the loop top. The oscillation period is consistent with the analytical result given by Eq. (26). For a first overtone transverse oscillation a blob initially near the loop top falls super-ballistically in the top half of the loop because of the additional downwards acceleration from the ponderomotive force. The blob has then picked up enough speed to continue to the photosphere.

We now consider the transverse oscillation damping time, $\tau$, to be finite. Then, an oscillation whose amplitude is above the critical value to keep a blob suspended, is not be able to keep the blob suspended indefinitely and once the amplitude drops below the critical value, the blob falls to the photosphere. Therefore, the number of turns the blob may complete in the phase diagram
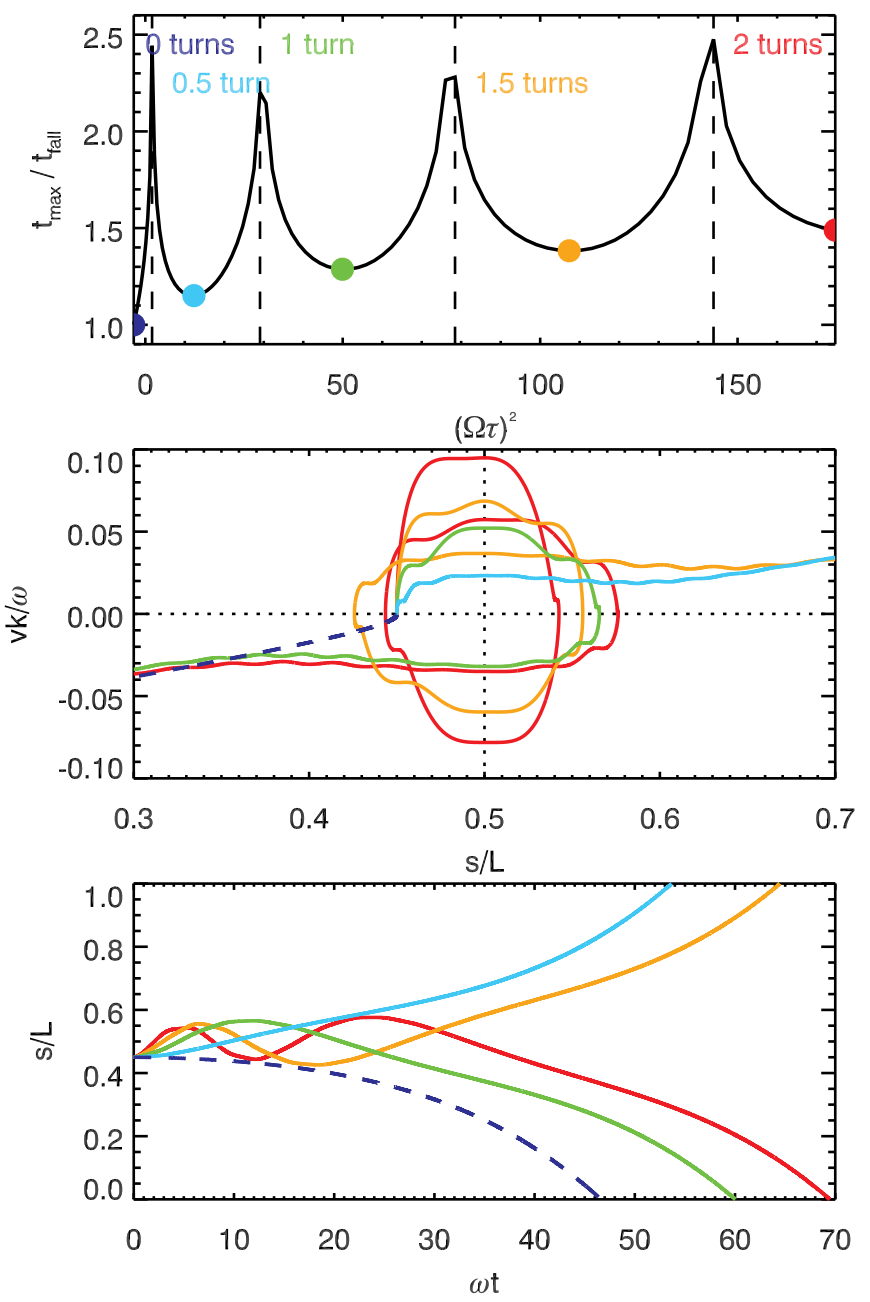

Fig. 3. Fall time $t_{\max }$ relative to ballistic time $t_{\text {fall }}$ as a function of $(\Omega \tau)^{2}$ (Top). Coloured dots indicate cases whose paths are shown in the phase space diagram (middle) and in the time-distance plot (bottom).

depends on the product $\Omega \tau$. Figure 3 illustrates the blob kinematics as a function of $\Omega \tau$. For a typical loop of radius $R=50 \mathrm{Mm}$, supporting a fundamental mode transverse loop oscillation with a period of $150 \mathrm{~s}$ and quality factor $\omega \tau=20$, we have $a_{\text {crit }}=0.08$. Then for a range of relative amplitude values $a=0.1,0.2,0.3$, we find values for $\Omega \tau=0.9,2.6$, and 4.1, respectively. In terms of periods $2 \pi / \Omega$, it corresponds to values of $56 \mathrm{~min}, 19 \mathrm{~min}$, and $12 \mathrm{~min}$, respectively.

\section{Modelling of observations of coronal rain oscillations}

We examine observations of coronal rain that exhibits nonmonotonic kinematics in the presence of transverse oscillations in NOAA AR 11461 on the north-east limb in the Ca II $H$ band of Hinode/SOT, taken on 16 April 2012 from 15:04 to 15:36 UT. The data has a spatial resolution of 0.22 arcsec and a time cadence of 24 s. Later from 17:24 UT onwards a large solar eruption takes place in the active region with an associated GOES class M1.7 flare that peaks at 17:45 UT. Transverse oscillations are excited in coronal loops in the vicinity. We examine one such loop in which coronal rain is present (see Fig. 4). We assume a semi-circular, planar model for the loop with a radius of $45 \mathrm{Mm}$ and an inclination of 30 degrees with respect to the photospheric 


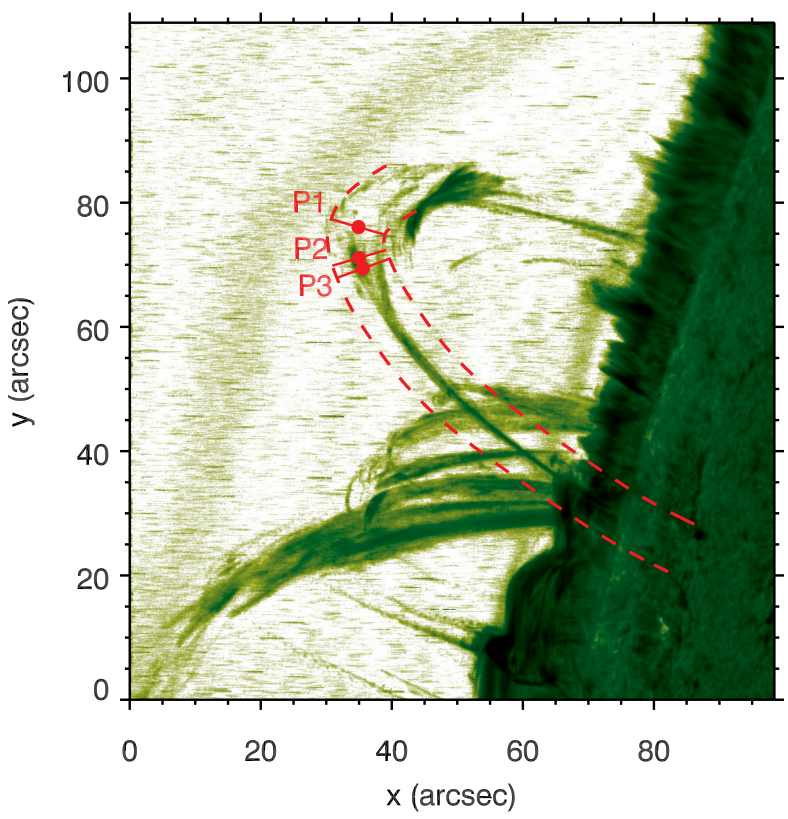

Fig. 4. Variance of Hinode/SOT intensity in the Ca II $H$ band on the north-east limb on 16 April 2012 for the time period between 15:04 and 15:36 UT. Regions of high variability are dark. The path along the oscillating loop is indicated by two parallel dashed lines.
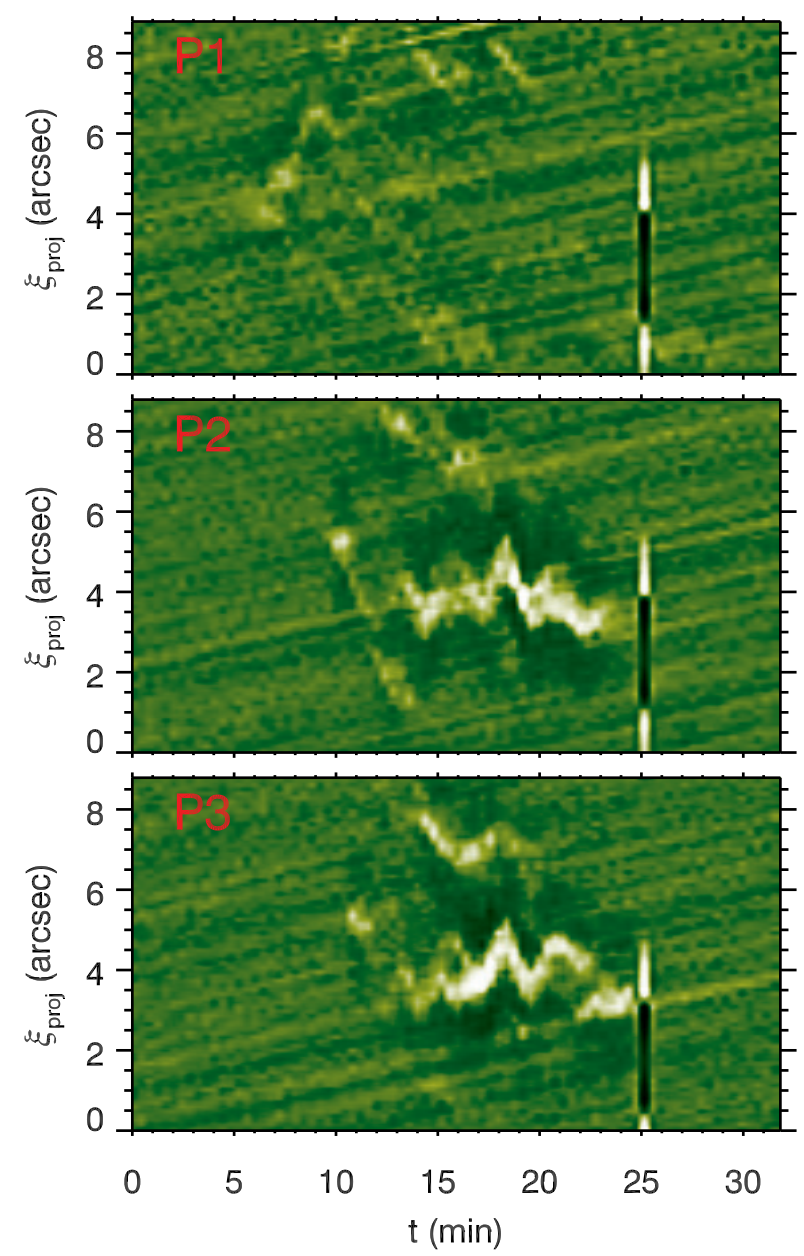

Fig. 5. Relative intensity as a function of time relative to 15:04 UT and distance across the path for three positions along the path indicated in Fig. 4.

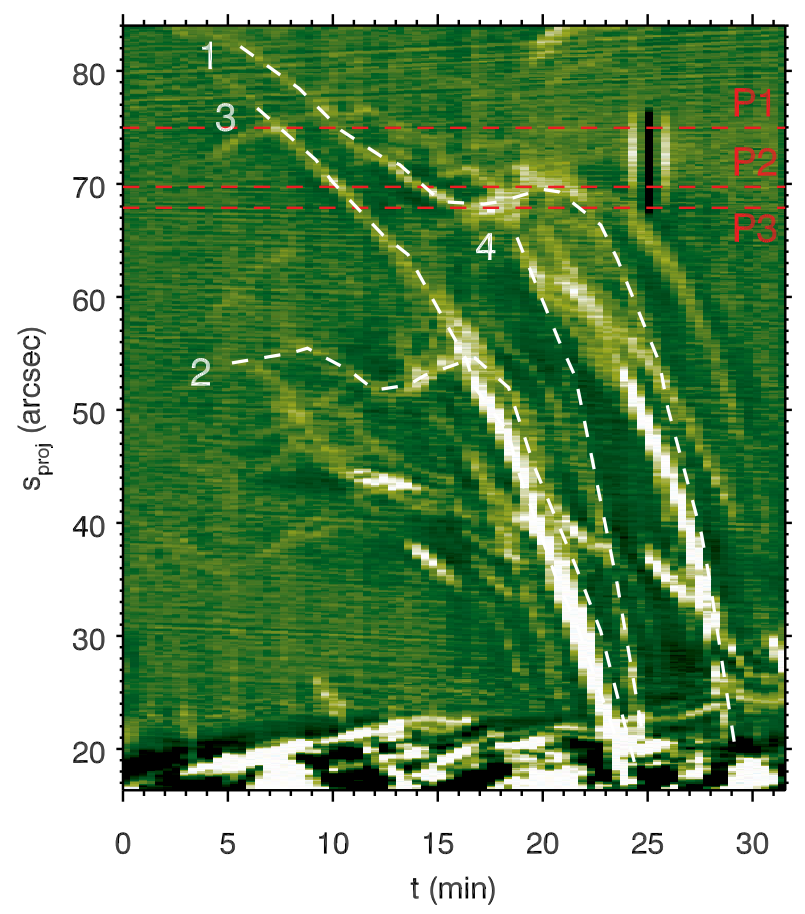

Fig. 6. Relative intensity as a function of time relative to 15:04 UT and distance along the path. The intensity has been averaged across part of the path width. The dashed lines show the parallel position of five coronal rain blobs as a function of time.

normal. The loop length is estimated assuming a semi-circular loop to be $L=140 \mathrm{Mm}$. It is reasonable to expect this loop length estimate to have a relative error of circa $20 \%$. A region of interest is selected along a loop path in which coronal rain is visible. Figure 5 shows the relative intensity across this path at three locations near the loop top. Several coronal rain blobs are seen to oscillate transversely with a period of approximately three minutes.

The oscillation characteristics are found by applying the same analysis method as used by Antolin \& Verwichte (2011). We observe the oscillation over approximately five periods. We find a mean oscillation period, $P=154 \pm 20 \mathrm{~s}$, and a mean transverse displacement amplitude, $\xi_{0}=1.1 \pm 0.4 \mathrm{Mm}$. From this follows a projected transverse velocity amplitude, $v_{\perp 0}=2 \pi \xi_{0} / P=$ $45 \pm 20 \mathrm{~km} \mathrm{~s}^{-1}$. The oscillation has the largest displacement near the loop top. We therefore assume that the loop is mainly oscillating in the fundamental harmonic mode. The wave number, $k=1 / R=0.022 \pm 0.01 \mathrm{Mm}^{-1}$. From this follows a relative oscillation amplitude $a=\xi_{0} k=0.025 \pm 0.01$.

Figure 6 shows the position of the coronal rain along the path as a function of time. Many coronal rain blobs are seen to be falling towards the solar surface non-monotonically. The blobs are seen to oscillate up and down a few times during their descent with a "longitudinal" period $P_{\text {long }}$ varying between 7 and $15 \mathrm{~min}$. Four coronal rain blobs are tracked in time as a function of distance along the loop. Figure 7 shows their positions as a function time and the position-velocity tracks in the phase diagram based on polynomial fits of the measurements. We focus on the coronal rain starting from near the loop top. We model the kinematics for a blob starting near the loop top for several initial speeds between -25 and $25 \mathrm{~km} \mathrm{~s}^{-1}$ and for the observed amplitude $a=0.025$. Also, a finite damping time, $\tau=280 \mathrm{~s}$, is included to allow us to model of rain falling non-monotonically. Figure 7 shows that the numerically generated kinematics shows 

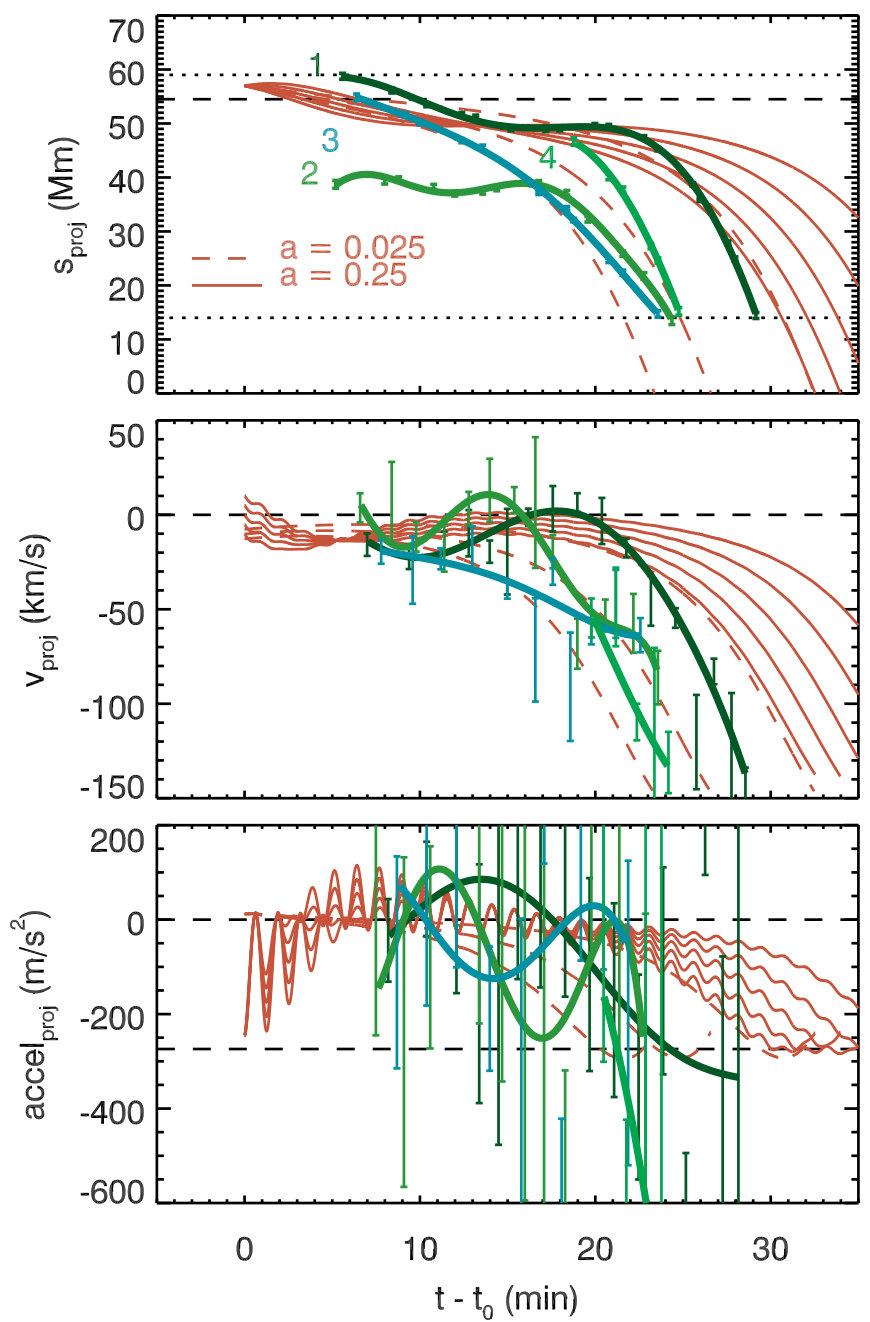

Fig. 7. Rain blob projected distance (top), speed (middle), and acceleration (bottom) as a function of time for five measured coronal rain blobs (error bars). The solid lines are polynomial fits to the measurements. The solid thin curves are numerically generated paths for blobs of negligible mass starting at $s=0.56 L$ for amplitude $a=0.25$ and initial parallel speeds of $-20,-10,0,10$, and $20 \mathrm{~km} \mathrm{~s}^{-1}$, respectively. The dashed thin curves are the equivalent for $a=0.025$ and speeds $-25,-20$, and $-15 \mathrm{~km} \mathrm{~s}^{-1}$. Time is measured relative to start time of the simulation.

reasonable agreement with the observations in terms of fall times and speeds. But, importantly, to explain a longitudinal periodicity of 15 min for observed rain \#1, i.e. $\Omega=7 \times 10^{-3} \mathrm{rad} \mathrm{s}^{-1}$, we require a larger oscillation amplitude. Using Eq. (26), with $g_{\odot}=274 \mathrm{~m} \mathrm{~s}^{-2}$, we estimate a value of $a=0.26\left(\xi_{0}=11 \mathrm{Mm}\right)$ is required to explain this periodicity. Figures 7 and 8 show that a good comparison is found for $a=0.25$ and $v_{0}=-25 \mathrm{~km} \mathrm{~s}^{-1}$. For the other initial velocities, the fall time is delayed by up to $30 \%$ compared with the observation of \#1.

We consider two further observational studies of rain kinematics in the presence of transverse oscillations by Antolin \& Verwichte (2011) and Kohutova \& Verwichte (2016), where rain does not exhibit non-monotonic kinematics, but we aim to model the sub-ballistic fall. Antolin \& Verwichte (2011) measured transverse rain oscillations with periods around $150 \mathrm{~s}$ and displacement amplitudes around $350 \mathrm{~km}$ in a loop estimated to be about $80 \pm 15 \mathrm{Mm}$ long. We forward-model rain kinematics for such a loop supporting undamped oscillations for a range of oscillation amplitudes. The highest value (shown in red) corresponds to $0.98 a_{\text {crit }}$. Figure 9 shows the kinematics for the

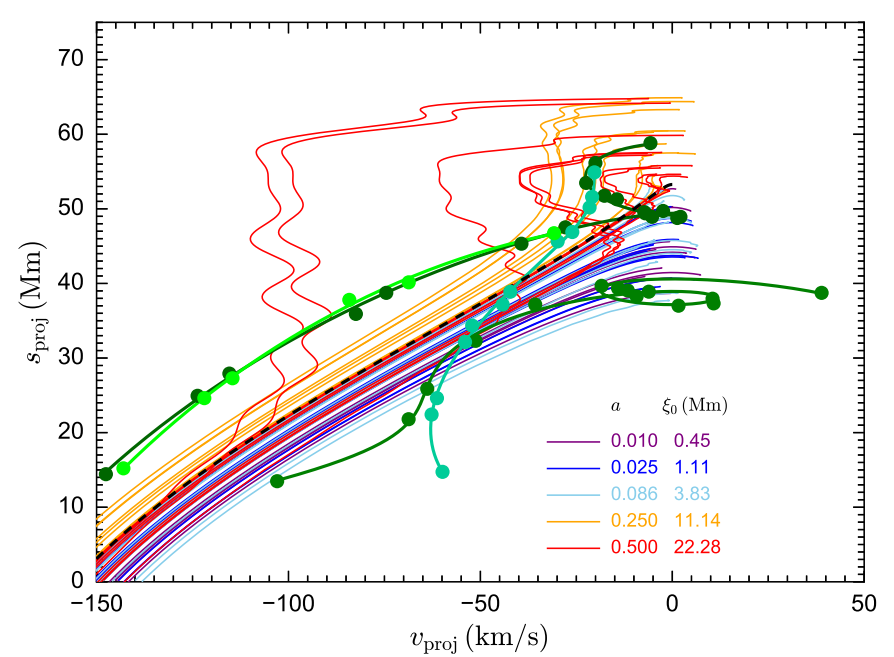

Fig. 8. Phase diagram of projected position vs. velocity for the 5 measured coronal rain blobs (in green). The solid green lines are polynomial fits to the measurements. The solid thin curves are numerically generated paths for blobs of negligible mass starting at random positions between $0.3 L$ and $0.65 L$ and speeds between $-25 \mathrm{~km} \mathrm{~s}^{-1}$ and $25 \mathrm{~km} \mathrm{~s}^{-1}$ for amplitudes ranging from $a=0.01$ to 0.5 .

projected speed and acceleration as a function of height for a loop of length $70 \mathrm{Mm}$. We chose a value of $70 \mathrm{Mm}$ instead of $80 \mathrm{Mm}$ as to be better able to directly compare with the results shown in Fig. 4 from Antolin \& Verwichte (2011). With this approach we ignored the effects of projection and non-circularity on the measurements. In order to match measured speeds of $30-40 \mathrm{~km} \mathrm{~s}^{-1}$, the oscillation displacement amplitude has to be larger than $2.5 \mathrm{Mm}$, which is at least seven times the observed amplitude and is larger than the critical amplitude for which the model predicts that the rain does not fall but is trapped around the loop top. We also show the effective downwards acceleration, i.e. the average projected acceleration along the path,

$\left\langle\frac{\mathrm{d} v}{\mathrm{~d} t}\right\rangle=\frac{2}{L} \int_{0}^{L / 2} \frac{\mathrm{d} v}{\mathrm{~d} t} \cos \left(\frac{\pi s}{L}\right) \mathrm{d} s=\frac{1}{T} \int_{0}^{T} v \frac{\mathrm{d} v}{\mathrm{~d} t} \cos \left(\frac{\pi s(t)}{L}\right) \mathrm{d} t$.

In the absence of the ponderomotive force, the effective acceleration becomes the effective gravity, which for a circular loop is of the form $\left\langle g_{\odot \text { eff }}\right\rangle=(2 / \pi) g_{\odot}=174 \mathrm{~m} \mathrm{~s}^{-2}$. As Fig. 9 shows, a finite oscillation amplitude lowers the effective acceleration from $\left\langle g_{\text {eff }}\right\rangle$ for $a=0$ to zero at $a=a_{\text {crit }}$. Antolin \& Verwichte (2011) measured an effective acceleration centred on a mean value of $56 \mathrm{~m} \mathrm{~s}^{-2}$. In order to reproduce this value an oscillation displacement within a narrow range around $2.5 \mathrm{Mm}$ is required.

Kohutova \& Verwichte (2016) measured oscillations in a loop of length $129 \mathrm{Mm}$ with periods around $200 \mathrm{~s}$ and displacement amplitudes of 200-400 km. From a similar analysis as performed for the previous observational study, we find that a displacement amplitude of at least $4 \mathrm{Mm}$, which is almost ten times the observed amplitudes, is required to explain the observations. Kohutova \& Verwichte (2016) also found long-period oscillation of periods around $17.4 \mathrm{~min}$ with a larger displacement of $1 \mathrm{Mm}$. However, since the main component of the ponderomotive force is proportional to $\omega^{2}$, the required amplitude to achieve the same uplift increases. Therefore, the same analysis performed for this periodicity shows that a displacement amplitude exceeding $20 \mathrm{Mm}$ is needed to explain the observed kinematics. 

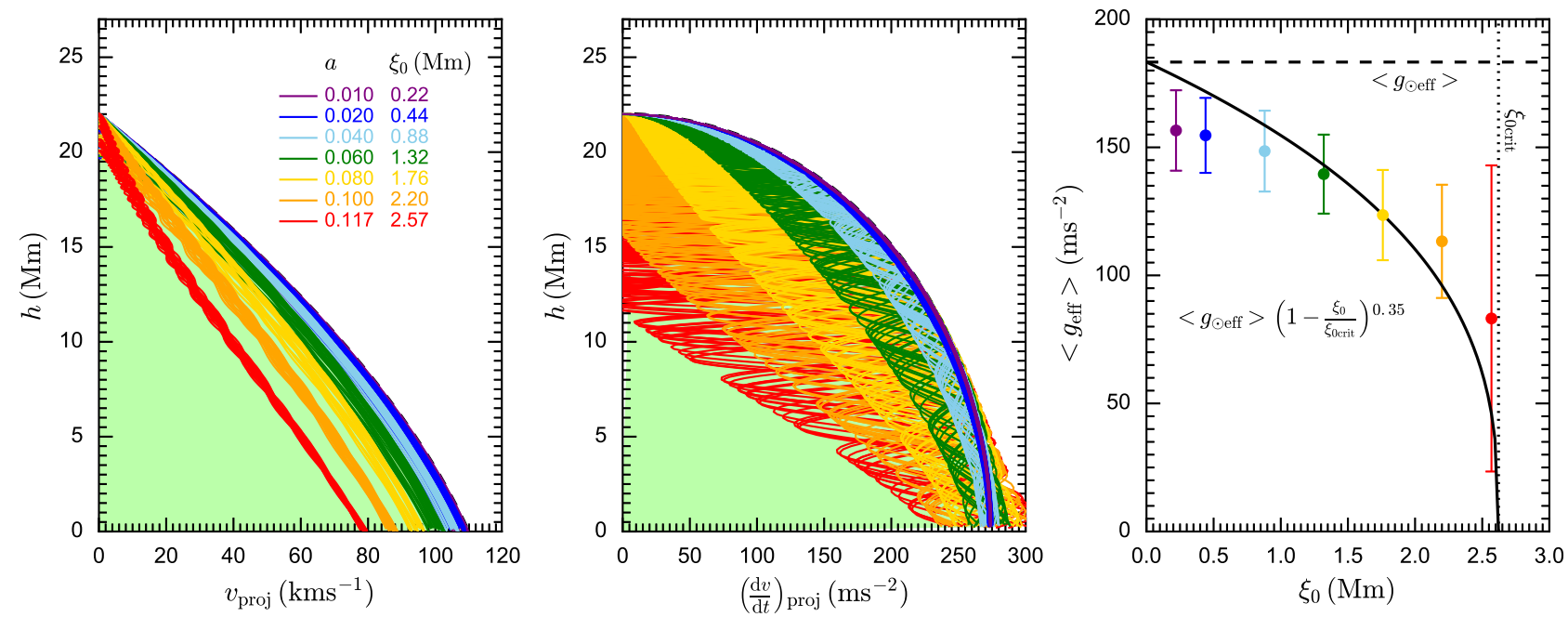

Fig. 9. Left and middle: simulated kinematics the height of rain blobs as a function of plane-of-the sky projected speed for a range of transverse oscillations amplitudes for a circular loop of length $70 \mathrm{Mm}$. Kinematic tracks for different amplitudes are represented by different colours. The green area represents the sub-ballistic regime. Right: effective acceleration as defined by Eq. (30) as a function of oscillation displacement amplitude. The effective gravity and critical displacement amplitude are highlighted. The solid curve is a best power-law fit within the constraints that it is equal to $\left\langle g_{\odot \text { eff }}\right\rangle$ for $a=0$ and 0 for $a=a_{\text {crit }}$.

\section{Excitation of transverse loop oscillations by coronal rain}

We may extend the model to include an excitation mechanism for transverse oscillations by coronal rain of finite mass, i.e. $\mu$ finite. There are several possible excitation scenarios. First, through the curvature of the loop, the inertia of the rain creates a centrifugal acceleration that moves the coronal loop radially outwards. We expect predominately that the first overtone of a vertically polarised oscillation is excited. Since the mechanical model does not include curved geometry, we do not examine this scenario here. Second, through the gravitational acceleration of the rain mass, concentration on a coronal loop moves the loop downwards. The polarisation of the mode depends on the inclination of the loop with respect to the photospheric normal. We again expect again a vertically polarised mode in an uninclined loop. But with finite inclination the rain mass also moves the coronal loop sideways along with downwards. For a rain blob starting near the loop top, we then expect predominately that the horizontally polarised fundamental mode is excited. We can easily model the second scenario by extending the model to include a loop that is inclined by an angle $\theta$ with respect to the photospheric normal. For such a loop, the gravity vector may be decomposed into three components, i.e.

$$
\begin{aligned}
\boldsymbol{g}(s) & =-g_{\odot} \mathbf{1}_{z} \\
& =-g_{\odot} \cos \theta\left[\sin \left(\frac{s}{R}\right) \mathbf{1}_{\perp}+\cos \left(\frac{s}{R}\right) \mathbf{1}_{\|}\right]-g_{\odot} \sin \theta \mathbf{1}_{\wedge}
\end{aligned}
$$

where $\mathbf{1}_{\|}, \mathbf{1}_{\perp}$ and $\mathbf{1}_{\wedge}$ form a coordinate triad and point radially and azimuthally in the loop plane, and normal to the plane, respectively (see Fig. 10). The governing wave equation for horizontally and vertically polarised oscillations become

$$
\begin{aligned}
& \mathcal{L} \xi_{\wedge}=-\mu L\left[\frac{\mathrm{d}^{2} \xi_{\curlywedge}}{\mathrm{d} t^{2}}+g_{\odot} \sin \theta\right] \delta\left(s-s_{\mathrm{p}}\right), \\
& \mathcal{L} \xi_{\perp}=-\mu L\left[\frac{\mathrm{d}^{2} \xi_{\perp}}{\mathrm{d} t^{2}}+g_{\odot} \cos \theta \sin \left(\frac{s}{R}\right)\right] \delta\left(s-s_{\mathrm{p}}\right),
\end{aligned}
$$

where

$$
\mathcal{L}=\frac{\partial^{2}}{\partial t^{2}}+\frac{2}{\tau} \frac{\partial}{\partial t}-V_{\mathrm{A}}^{2} \frac{\partial^{2}}{\partial s^{2}},
$$

and $\xi_{\wedge}$ and $\xi_{\perp}$ are the horizontally and vertically polarised displacements. respectively. Equation (32) is turned into a system of ordinary differential equations for each polarisation using a decomposition into spatial harmonics that satisfy the boundary conditions,

$\xi(s, t)=\sum_{n=1}^{\infty} \xi_{n}(t) \sin \left(\frac{n s}{R}\right)$.

The delta function is likewise decomposed into

$\delta\left(s-s_{\mathrm{p}}\right)=\frac{2}{L} \sum_{n=1}^{\infty} \sin \left(\frac{n s_{\mathrm{p}}}{R}\right) \sin \left(\frac{n s}{R}\right)$.

Equation (32) is decomposed into the system of ODEs

$$
\begin{aligned}
& \mathcal{L}_{n} \xi_{\wedge n}=-2 \mu \sum_{k} \sin \left(\frac{k s_{\mathrm{p}}}{R}\right)\left[\sum_{l} c_{n, k, l} \frac{\mathrm{d}^{2} \xi_{\wedge, l}}{\mathrm{~d} t^{2}}+g_{\odot} \sin \theta \delta_{n, k}\right] \\
& \mathcal{L}_{n} \xi_{\perp, n}=-2 \mu \sum_{k} \sin \left(\frac{k s_{\mathrm{p}}}{R}\right)\left[\sum_{l} c_{n, k, l} \frac{\mathrm{d}^{2} \xi_{\perp, l}}{\mathrm{~d} t^{2}}+g_{\odot} \cos \theta c_{n, k, 1}\right],
\end{aligned}
$$

where

$$
\mathcal{L}_{n}=\frac{\mathrm{d}^{2}}{\mathrm{~d} t^{2}}+\frac{2}{\tau_{n}} \frac{\mathrm{d}}{\mathrm{d} t}+\left(\frac{V_{\mathrm{A}} n}{R}\right)^{2},
$$

and

$$
\begin{aligned}
c_{n, k, l} & =\frac{2}{L} \int_{0}^{L} \sin \left(\frac{n s}{R}\right) \sin \left(\frac{k s}{R}\right) \sin \left(\frac{l s}{R}\right) \mathrm{d} s \\
& =\hat{C}_{n+k-l}-\hat{C}_{n-k-l}+\hat{C}_{n-k+l}-\hat{C}_{n+k+l},
\end{aligned}
$$

with $\hat{C}_{p}=(\pi p)^{-1}$ if $p$ is odd and $\hat{C}_{p}=0$ if $p$ is even. Also, in keeping with the resonant absorption mechanism, $\tau_{n}=\tau_{1} / n$. 


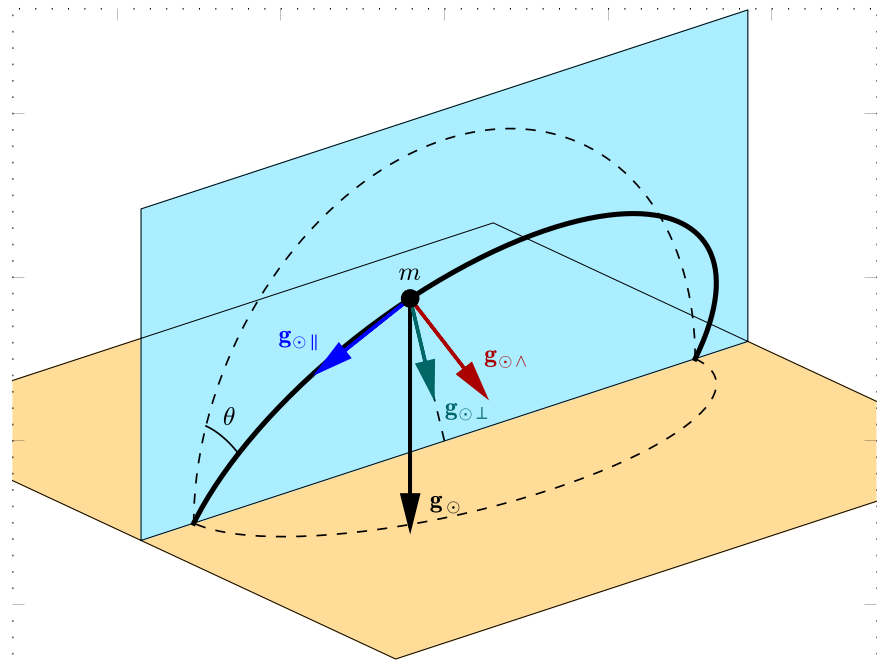

Fig. 10. Model of an inclined loop with the decomposition gravity vector at the rain blob, where $\boldsymbol{g}_{\odot, \|}=-g_{\odot} \cos \theta \cos \left(\frac{s}{R}\right) \mathbf{1}_{\|}, \boldsymbol{g}_{\odot, \perp}=$ $-g_{\odot} \cos \theta \sin \left(\frac{s}{R}\right) \mathbf{1}_{\perp}$ and $\boldsymbol{g}_{\odot, \wedge}=-g_{\odot} \sin \theta \mathbf{1}_{\wedge}$.

The system of ODEs (Eq. (37)), together with Eq. (6), are numerically integrated for $\xi_{n}(t)$ and the rain kinematics. To avoid an excitation of oscillations due to an instantaneous appearance of a coronal rain mass at $t=0$, we include a ramp up of the mass of the rain over a typical time duration of $t_{\text {ramp }}=100 \mathrm{~s}$, i.e. $m(t)=m_{0}\left(1-\exp \left(-t / t_{\text {ramp }}\right)\right)$. In reality, the blob excites in both horizontal and vertical modes. For simplicity, in the modelling presented here we ignored the coupling between the two polarisation through the influence of the ponderomotive force on the blob kinematics.

Figure 11 shows an example of the excitation of a transverse oscillation by the coronal rain by solving Eqs. (6) and (37) numerically for a typical coronal rain blob with mass $m / M=0.2$ $(\mu=0.25)$. It confirms that predominately the fundamental harmonic is excited. The blob is displaced down from the initial position with a superimposed oscillation. The displacement amplitude of the generated oscillation is of the order of $0.1-0.2 \%$ of loop length. For a loop of $100 \mathrm{Mm}$ length, this corresponds to an amplitude of the order of hundreds of $\mathrm{km}$. The exact value depends on the choice of damping time $\tau_{1}$ and ramp-up time $t_{\text {ramp }}$, where the largest amplitudes are found where these times are equal to infinity and zero.

Figure 12 shows that the maximum displacement amplitude $\xi_{1}$, for a rain blob that falls from an initially stationary position near the loop top at $0.475 L$, is approximately linearly proportional to the coronal rain mass $m / M$ and $\sqrt{\theta}$, i.e.

$$
\begin{aligned}
& \xi_{1, \wedge}=(9.6 \pm 0.7) \times 10^{-3} \sqrt{\frac{2 \theta}{\pi}} \frac{m}{M} L \\
& \xi_{1, \perp}=(5.0 \pm 0.6) \times 10^{-3} \sqrt{1-\frac{2 \theta}{\pi}} \frac{m}{M} L .
\end{aligned}
$$

The exact values depend on the initial conditions, i.e. position and speed, of the rain blob, and decrease as the starting position moves down a loop leg. The vertically polarised mode has half the amplitude of the horizontally polarised mode. The displacement amplitude is of the order of several hundreds of $\mathrm{km}$. This is roughly consistent with observed rain displacement amplitudes. Furthermore, when the displacement amplitude of a rain blob or shower is measured, the rain kinematics is understood, and the
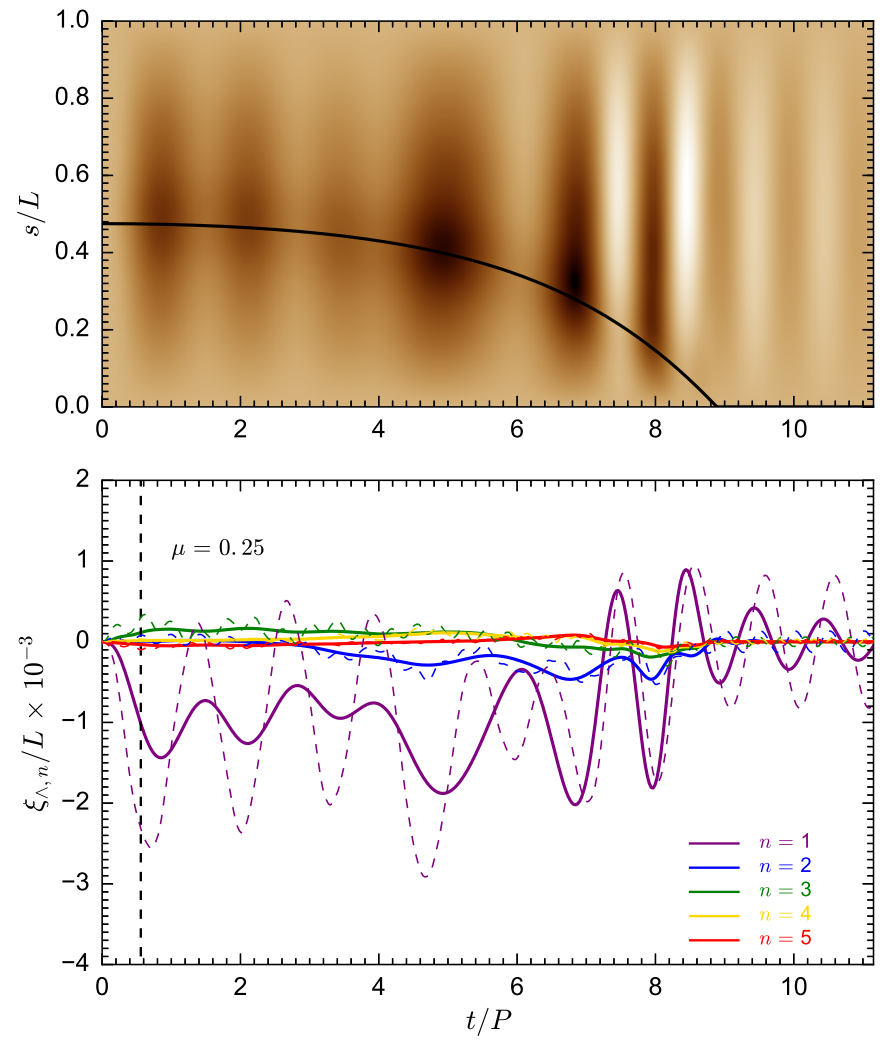

Fig. 11. Top: horizontally polarised wave field $\xi_{\wedge}$ as a function of time and space for a coronal rain blob forming at $s=0.48 \mathrm{~L}$ with a mass corresponding to $\mu=0.25$. The loop has length $100 \mathrm{Mm}$, Alfvén speed $V_{\mathrm{A} 0}=1000 \mathrm{~km} \mathrm{~s}^{-1}$, and an inclination of $\theta=50^{\circ}$. Also, $\tau_{1}=500 \mathrm{~s}$ and $t_{\mathrm{ramp}}=100 \mathrm{~s}$. The curve is the path $s_{\mathrm{p}}(t)$. Bottom: time evolution of the 5 first harmonic amplitudes $\xi_{\wedge, n}$. The dashed curves are the equivalent for $\tau_{1} \rightarrow \infty$ and $t_{\mathrm{ramp}}=0 \mathrm{~s}$.

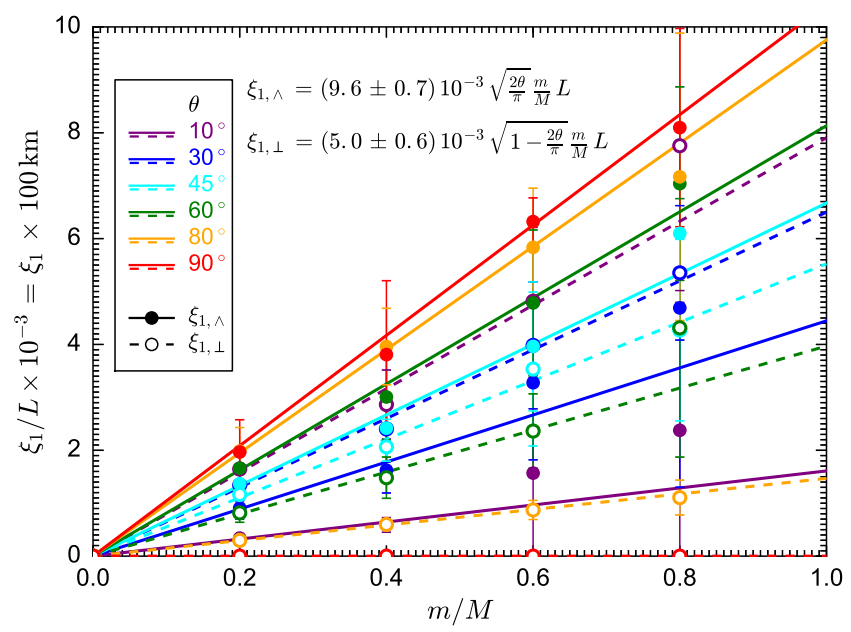

Fig. 12. Oscillation amplitude of the fundamental harmonic of a horizontally and vertically polarised transverse oscillation as a function of coronal rain mass $m / M$ for the same loop as in Fig. 11 for several loop inclination angles and with $\tau_{1} \rightarrow \infty$ and $t_{\text {ramp }}=0 \mathrm{~s}$. Initially the rain blob is located at $0.745 \mathrm{~L}$ with zero speed.

loop inclination is known, a seismological method is available to estimate the fraction of the rain mass relative to the hot loop mass.

In Fig. 13 the Eqs. (39) are applied to the three previously examined observational studies, i.e. event of 16 April 2012, 

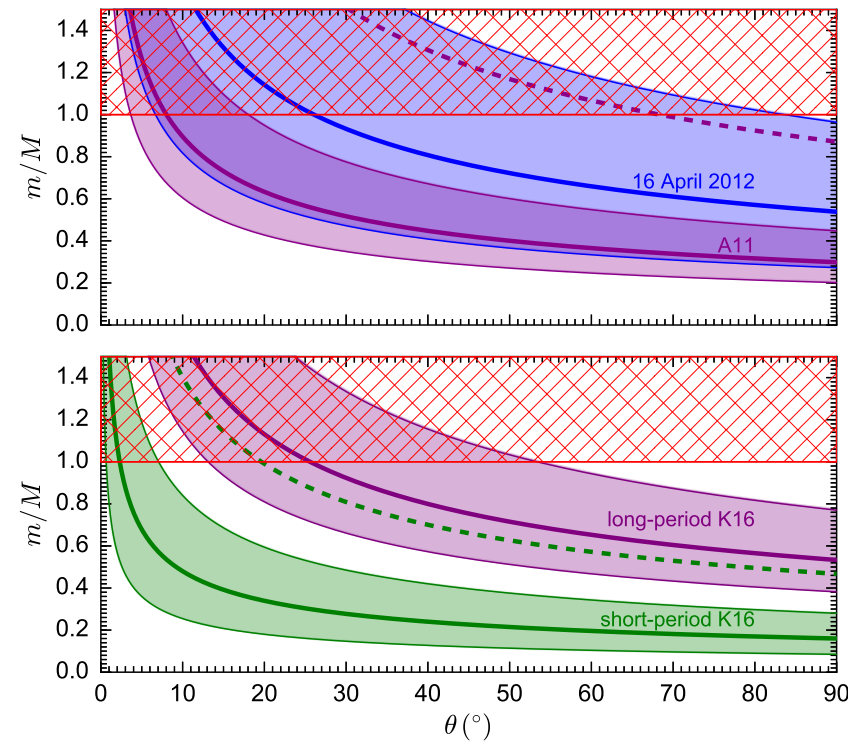

Fig. 13. Rain mass ratio $m / M$ as a function of loop inclination $\theta$ by applying Eqs. (39) to the three discussed observational studies. Thick solid (dashed) lines are for horizontally (vertically) polarised modes. The shaded areas are the one-sigma errors.

Table 1. Event parameters.

\begin{tabular}{l|rrc}
\hline \hline Event & \multicolumn{1}{|c}{$L(\mathrm{Mm})$} & \multicolumn{1}{c}{$\xi_{0}(\mathrm{~km})$} & $\xi_{0} 10^{3} / L$ \\
\hline 16 April 2012 & $140 \pm 28$ & $1100 \pm 400$ & $8 \pm 6$ \\
A11 & $80 \pm 15$ & $350 \pm 60$ & $4 \pm 2$ \\
Short-period K16 & $128 \pm 30$ & $300 \pm 100$ & $2 \pm 2$ \\
Long-period K16 & $128 \pm 30$ & $1000 \pm 100$ & $8 \pm 3$ \\
\hline
\end{tabular}

studies by Antolin \& Verwichte (2011, hereafter A11) and Kohutova \& Verwichte (2016, hereafter K16) using the relevant parameters as shown in Table 1 . We assume that the simulated rain blob represents a rain shower. In all these studies the examined loops show small inclinations with respect to the photospheric normal of $30^{\circ}$ or less. The smallamplitude horizontally polarised transverse oscillations observed by Kohutova \& Verwichte (2016) could be explained by wave excitation provided the rain mass comprises at least $20-40 \%$ of the total loop mass. The oscillations observed by Antolin \& Verwichte (2011) could marginally be explained by wave excitation but the rain mass would have to comprise at least $60-80 \%$ of the total loop mass. The event of 16 April 2012 and the long-period oscillations seen by Kohutova \& Verwichte (2016) can be ruled out to be excited by the rain because they would require a rain mass that is unrealistically high, i.e. close to or even exceeding $100 \%$ of the total loop mass.

\section{Discussion}

We have studied the kinematics of coronal rain in the presence of a transversely oscillating loop using an analytical model of a rain blob guided by the coronal magnetic field as the mechanical analogue of a moving point mass on an oscillating string with gravity. The ponderomotive force from the shear oscillation pushes rain blobs towards the anti-nodes in their displacement field. A fundamental mode creates an additional force besides gravity acting on coronal rain towards the loop top. The fall of coronal rain is thus becoming sub-ballistic. If a critical oscillation amplitude is exceeded, the rain does not fall at all but oscillates slowly up and down around the loop top. If the oscillation is damped, this motion is temporary and as soon as the amplitude goes below the critical amplitude, the rain eventually falls towards the surface. We sought to identify these characteristics in several observational studies of coronal rain in the presence of transverse oscillations. We found that up-and-down motions of coronal rain is indeed seen. In one study we found that though we can explain the observed rain speeds, the amplitude necessary to explain the long periodicity is about ten times larger than what is observed. It is not likely that this discrepancy can be understood by a line-of-sight effect of the plane of polarisation of the oscillation. We investigated two further observational studies where sub-ballistic rain is seen in the presence of oscillations. We found that the required amplitude also tends to be about an order of magnitude higher than what is observed. Therefore, the ponderomotive force is not likely to be the primary physical reason for sub-ballistic fall of coronal rain in most cases. But this is not surprising since sub-ballistic motion has been observed in the absence of any oscillations. This study, however, allows us to evaluate the contribution from the ponderomotive force on coronal rain kinematic.

The model also allows us to study the excitation of transverse oscillations by the presence of the coronal rain mass in a loop under the influence of solar gravity. The appearance of a cold localised mass displaces the loop downwards. This sets up a transverse oscillation in the loop. Its amplitude depends on the mass ratio between rain and hot loop plasma, the inclination of the loop with respect to the photospheric normal, the timescale at which the rain forms, and the inertia. The fundamental mode is predominately excited with predicted displacement amplitudes in the range of hundreds of $\mathrm{km}$ for rain forming near the loop top. This is in the range of those often observed. In fact, we may explain the short-period oscillations reported by Kohutova \& Verwichte (2016) as rain-excited provided the rain mass comprises at least $20-40 \%$ of the total loop mass. For other events, the required rain mass may be too high or impossible. However, Antolin et al. (2015) have shown that in some cases rain showers may make up most of the mass of the loop. Also, the vertically polarised mode has a predicted amplitude that is half that of a horizontally polarised mode (at $\theta=45^{\circ}$ ). The presence of coronal rain in loops may be an important mechanism for exciting transverse oscillations.

The analytical model is limited in that it does not take the roles of plasma thermodynamics, pressure, or transverse structuring into account. Detailed numerical nonlinear MHD simulations will be undertaken to obtain a fuller picture of the relationship between transverse oscillations and coronal rain. However, this study will help to recognise those features in the simulations arising from the ponderomotive action or wave excitation.

Acknowledgements. E.V. acknowledges support from the Warwick STFC Consolidated Grant ST/L000733/I. P.A. acknowledges support from the EU Horizon 2020 Research and Innovation programme (grant agreement No. 647214). P.K. acknowledges support from a UK STFC Ph.D. studentship. T.N. acknowledges support from the St Andrews STFC Consolidated Grant SN/N000609/1. Hinode is a Japanese mission developed and launched by ISAS/JAXA, with NAOJ as domestic partner and NASA and STFC (UK) as international partners. It is operated by these agencies in co-operation with ESA and NSC (Norway). The work was also supported by the international programme "Implications for coronal heating and magnetic fields from coronal rain observations and modelling" of the International Space Science Institute (ISSI), Bern. This research has made use of SunPy, an open-source and free community-developed solar data analysis package written in Python (http://sunpy.org).

\section{References}

Ahn, K., Chae, J., Cho, K.-S., et al. 2014, Sol. Phys., 289, 4117

Allan, W. 1993, J. Geophys. Res., 98, 1409 
Allan, W., \& Manuel, J. R. 1996, Annales Geophysicae, 14, 893

Andries, J., Van Doorsselaere, T., Roberts, B., et al. 2009, Space Sci. Rev., 149, 3 Anfinogentov, S. A., Nakariakov, V. M., \& Nisticò, G. 2015, A\&A, 583, A136 Antolin, P., \& Rouppe van der Voort, L. 2012, ApJ, 745, 152

Antolin, P., \& Verwichte, E. 2011, ApJ, 736, 121

Antolin, P., Shibata, K., \& Vissers, G. 2010, ApJ, 716, 154

Antolin, P., Vissers, G., Pereira, T. M. D., Rouppe van der Voort, L., \& Scullion, E. $2015, \mathrm{ApJ}, 806,81$

Arregui, I., \& Asensio Ramos, A. 2011, ApJ, 740, 44

Aschwanden, M. J., \& Schrijver, C. J. 2011, ApJ, 736, 102

Aschwanden, M. J., Fletcher, L., Schrijver, C. J., \& Alexander, D. 1999, ApJ, 520,880

Aschwanden, M. J., de Pontieu, B., Schrijver, C. J., \& Title, A. M. 2002, Sol. Phys., 206, 99

Athay, R. G., White, O. R., Lites, B. W., \& Bruner, Jr., E. C. 1980, Sol. Phys., 66,357

De Groof, A., Berghmans, D., van Driel-Gesztelyi, L., \& Poedts, S. 2004, A\&A, 415,1141

De Groof, A., Bastiaensen, C., Müller, D. A. N., Berghmans, D., \& Poedts, S. 2005, A\&A, 443, 319

De Moortel, I., \& Brady, C. S. 2007, ApJ, 664, 1210

De Pontieu, B., Title, A. M., Lemen, J. R., et al. 2014, Sol. Phys., 289, 2733

Fang, X., Xia, C., \& Keppens, R. 2013, ApJ, 771, L29

Fang, X., Xia, C., Keppens, R., \& Van Doorsselaere, T. 2015, ApJ, 807, 142

Goossens, M., Terradas, J., Andries, J., Arregui, I., \& Ballester, J. L. 2009, A\&A, 503,213

Goossens, M., Soler, R., Arregui, I., \& Terradas, J. 2012, ApJ, 760, 98

Hershaw, J., Foullon, C., Nakariakov, V. M., \& Verwichte, E. 2011, A\&A, 531, A53

Kawaguchi, I. 1970, PASJ, 22, 405

Kleint, L., Antolin, P., Tian, H., et al. 2014, ApJ, 789, L42

Klimchuk, J. A., Patsourakos, S., \& Cargill, P. J. 2008, ApJ, 682, 1351

Kohutova, P., \& Verwichte, E. 2016, ApJ, 827, 39

Leroy, J.-L. 1972, Sol. Phys., 25, 413

Levine, R. H., \& Withbroe, G. L. 1977, Sol. Phys., 51, 83

Luna, M., Karpen, J. T., \& DeVore, C. R. 2012, ApJ, 746, 30

Mackay, D. H., \& Galsgaard, K. 2001, Sol. Phys., 198, 289

McIntosh, S. W., de Pontieu, B., Carlsson, M., et al. 2011, Nature, 475, 477

Mrozek, T. 2011, Sol. Phys., 270, 191

Müller, D. A. N., Hansteen, V. H., \& Peter, H. 2003, A\&A, 411, 605

Müller, D. A. N., De Groof, A., Hansteen, V. H., \& Peter, H. 2005, A\&A, 436, 1067

Nakariakov, V. M., \& Ofman, L. 2001, A\&A, 372, L53
Nakariakov, V. M., Ofman, L., Deluca, E. E., Roberts, B., \& Davila, J. M. 1999, Science, 285, 862

Nayfeh, A. 2004, Perturbation Methods (John Wiley and Sons)

Nisticò, G., Nakariakov, V. M., \& Verwichte, E. 2013, A\&A, 552, A57

Oliver, R., Soler, R., Terradas, J., Zaqarashvili, T. V., \& Khodachenko, M. L. 2014, ApJ, 784, 21

Pascoe, D. J., Hood, A. W., De Moortel, I., \& Wright, A. N. 2013, A\&A, 551, A40

Ruderman, M. S., \& Roberts, B. 2002, ApJ, 577, 475

Scharmer, G. B., Bjelksjo, K., Korhonen, T. K., Lindberg, B., \& Petterson, B 2003, in SPIE Conf. Ser. 4853, eds. S. L. Keil, \& S. V. Avakyan, 341

Scharmer, G. B., Narayan, G., Hillberg, T., et al. 2008, ApJ, 689, L69

Schrijver, C. J. 2001, ApJ, 547, 475

Straus, T., Fleck, B., \& Andretta, V. 2015, A\&A, 582, A116

Terradas, J., \& Ofman, L. 2004, ApJ, 610, 523

Threlfall, J., De Moortel, I., McIntosh, S. W., \& Bethge, C. 2013, A\&A, 556, A124

Tomczyk, S., McIntosh, S. W., Keil, S. L., et al. 2007, Science, 317, 1192

Tsuneta, S., Ichimoto, K., Katsukawa, Y., et al. 2008, Sol. Phys., 249, 167

Van Doorsselaere, T., Nakariakov, V. M., \& Verwichte, E. 2007, A\&A, 473, 959

Van Doorsselaere, T., Nakariakov, V. M., \& Verwichte, E. 2008a, ApJ, 676, L73

Van Doorsselaere, T., Nakariakov, V. M., Young, P. R., \& Verwichte, E. 2008b, A\&A, 487, L17

Vashalomidze, Z., Kukhianidze, V., Zaqarashvili, T. V., et al. 2015, A\&A, 577, A136

Verth, G., Terradas, J., \& Goossens, M. 2010, ApJ, 718, L102

Verwichte, E. 1999, Ph.D. Thesis, The Open University

Verwichte, E., Nakariakov, V. M., \& Longbottom, A. W. 1999, J. Plasma Phys., 62, 219

Verwichte, E., Nakariakov, V. M., Ofman, L., \& Deluca, E. E. 2004, Sol. Phys., 223, 77

Verwichte, E., Aschwanden, M. J., Van Doorsselaere, T., Foullon, C., \& Nakariakov, V. M. 2009, ApJ, 698, 397

Verwichte, E., Foullon, C., \& Van Doorsselaere, T. 2010, ApJ, 717, 458

Verwichte, E., Van Doorsselaere, T., Foullon, C., \& White, R. S. 2013a, ApJ, 767, 16

Verwichte, E., Van Doorsselaere, T., White, R. S., \& Antolin, P. 2013b, A\&A, 552, A138

Wang, T. J., \& Solanki, S. K. 2004, A\&A, 421, L33

Wang, T., Ofman, L., Davila, J. M., \& Su, Y. 2012, ApJ, 751, L27

White, R. S., \& Verwichte, E. 2012, A\&A, 537, A49

White, R. S., Verwichte, E., \& Foullon, C. 2012, A\&A, 545, A129

White, R. S., Verwichte, E., \& Foullon, C. 2013, ApJ, 774, 104 